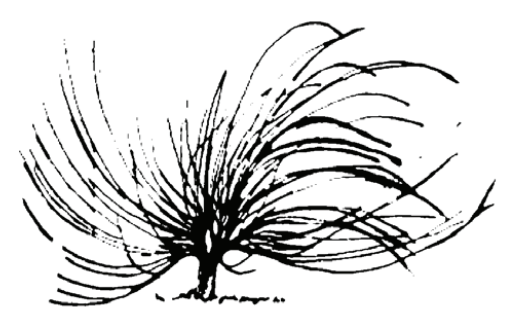

\title{
Cartografías Curriculares e Imaginarios de la Docencia Universitaria: El caso de la Maestría en Educación con Énfasis en Docencia Universitaria de la División de Educología del CIDE
}

\author{
Andrés Mora Ramírez ${ }^{1}$ \\ Universidad Nacional \\ Heredia, Costa Rica \\ andres.mora.ramirez@una.cr
}

\section{Resumen}

Este ensayo presenta y discute los principales hallazgos de una investigación acerca del Plan de Estudios de la Maestría en Educación con énfasis en Docencia Universitaria de la División de Educología del CIDE, realizada desde una perspectiva de análisis crítico sociocultural, en clave latinoamericana. Si bien el posgrado ha realizado una contribución valiosa a la profesionalización de docentes universitarios, el estudio encontró que el Plan de Estudios sufre de una serie de indefiniciones a nivel de sus opciones filosóficas, pedagógicas y epistemológicas, así como en la caracterización de su objeto de estudio; lo anterior, debido a la ausencia reconstrucciones curriculares profundas a lo largo de sus más de 15 años de vigencia. En este sentido, se recomienda que en la formulación

\section{(c) (i) $($ ) $\Theta$}

Recibido: 3 de agosto de 2015-Aprobado: 4 de diciembre de 2015

1 Profesor e investigador en el Instituto de Estudios Latinoamericanos y el Centro de Investigación y Docencia en Educación - División de Educología, ambas instancias de la Universidad Nacional (UNA). Magíster en Estudios Latinoamericanos con énfasis en Cultura y Desarrollo y Magíster en Educación con énfasis en Docencia Universitaria, títulos obtenidos en la UNA. 
de un nuevo diseño curricular, que responda a las expectativas de la sociedad y la docencia universitaria del presente, se incorporen también las perspectivas pedagógicas derivadas del pensamiento crítico latinoamericano.

Palabras clave: docencia universitaria, diseño curricular, ciencias de la educación, pedagogía

\begin{abstract}
This essay describes presents and discusses the main findings of a research project on the curriculum of the master's degree in Education with a concentration in higher education of the "Division de Educologia del CIDE;" this research was carried out from a Latin American perspective of socio-cultural critical analysis. Although the postgraduate has made a valuable contribution to the professionalization of university faculty, the study found that the curriculum is suffering from a series of uncertainties at the level of its philosophical, pedagogical, and epistemological options as well as the characterization of its subject matter, all of which are due to the absence of deep curricular reconstructions throughout its more than 15 years of existence. In this sense, it is recommended to consider the formulation of a new curriculum that meets the current expectations of society and incorporates the perspectives of the Latin American pedagogical thinking.
\end{abstract}

Keywords: university teaching, curriculum design, educational sciences and pedagogy

\title{
Introducción: puntos de partida $\mathbf{y}$ precisiones teóricas $\mathbf{y}$ metodológicas
}

Toda experiencia de investigación implica un esfuerzo dialéctico y de reconstrucción de aprendizajes y saberes, que sobrepasan al propio investigador y que, en consecuencia, no pueden limitarse al simple ejercicio academicista de su puesta en papel o en formatos digitales, muchas veces condenados a morir en el ostracismo de las bibliotecas o los repositorios digitales. Por el contrario, los nuevos hallazgos deben ser puestos en circulación para que, en ese viaje, propicien discusiones, 
reflexiones, debates y nuevas lecturas que animen los itinerarios académicos y curriculares de los centros de formación de docentes. Como bien decía el intelectual costarricense Joaquín García Monge, "si andan, las ideas crecen, triunfan" (Garrón, 1971, p. 156). Andando y creciendo en el quehacer colectivo, los nuevos saberes pueden echar raíces profundas y enriquecer nuestras prácticas con perspectiva de futuro.

En esa tesitura, este texto pretende socializar los hallazgos y vivencias de una investigación realizada en el Centro de Investigación y Docencia en Educación (CIDE) de la Universidad Nacional de Costa Rica (UNA), entre los años 2012 y 2013, cuyo propósito fue realizar una lectura crítica del Plan de Estudios de la Maestría en Educación con énfasis en Docencia Universitaria, programa de posgrado adscrito a la División de Educología del CIDE (Mora, 2014). La creación de esta Maestría en 1998 constituyó un hito importante, toda vez que por entonces la UNA experimentaba fuertes presiones y restricciones presupuestarias, además, la educación superior sufría el impacto de las reformas educativas neoliberales que recorrían todo el continente.

Uno de los informantes que colaboró en este proyecto, y que participó activamente en la construcción y redacción del primer Plan de Estudios, destacó el liderazgo y la visión que tuvo en aquellos días la Dra. Iza Vicenzi Gang, al optar por una mención que se engarzaba con la función que, desde el Estatuto Orgánico entonces vigente, se le asignaba al CIDE, a saber: la de desarrollar un programa de capacitación docente en didáctica universitaria para las distintas facultades y centros, y que por distintas razones políticas y administrativas, se había perdido. "El énfasis en Docencia Universitaria era una forma de decir ipresente! en el ámbito de la educación superior estatal" (C. Lépiz, comunicación personal, 8 de octubre, 2013).

Para llevar adelante la indagación se construyó un andamiaje teórico con fuerte acento sociocultural, que tuvo como principales referentes conceptuales la teoría sociológica de la producción de las ausencias -que confronta el carácter excluyente de la modernidad y sus regulacionesde Boaventura de Sousa Santos (2006); las ideas del filósofo argentino Arturo Roig (2009), sobre los olvidos como estrategia hegemónica en la constitución del sujeto social latinoamericano -y que aquí extendemos, también, a la formación de docentes-; y por supuesto, la perspectiva de interpretación crítica del currículo, del chileno Rolando Pinto (2008, 2009, 2012). Todos estos enfoques se complementaron con aportes vivenciales 
y reflexivos de las personas entrevistadas, quienes ayudaron a ampliar el horizonte de comprensión del problema de estudio.

La investigación partió de la premisa según la cual un Plan de Estudios, como todo diseño curricular, es un texto, un discurso con sus propios registros, en el que es posible identificar e interpretar sus condiciones de producción y recepción (contexto), su intencionalidad educativa (racionalidad curricular) y su intencionalidad ideológica (concepciones subyacentes, visiones sobre la docencia, el docente y su función en la sociedad, etc.). Todo lo cual permite caracterizar su formulación y su puesta en ejecución como un dispositivo cultural, como se discutió en un trabajo anterior (Mora, en prensa). Por medio de un instrumental metodológico hermenéutico, se efectuó un análisis de contenido cualitativo del documento del Plan de Estudios y otros documentos curriculares (rediseño curricular, programas de curso) en el período 1998-2011, con lo que se logró identificar los planteamientos y constructos significativos relacionados con las categorías analíticas y luego, a partir de esto, se interpretó su sentido dentro de la propuesta curricular en su conjunto y en su contexto de creación.

Antes de presentar los hallazgos, es necesario indicar que una de las principales dificultades con las que se enfrenta el lector crítico de los documentos del Plan de Estudios (la primera propuesta de 1998 y su rediseño del año 2009) es la ambigüedad de sus planteamientos y las debilidades de su estructura formal, en la que parece predominar un afán por limitarse a cumplir con los requisitos mínimos de aprobación por parte de las instancias administrativas de la UNA, pero sin ahondar en una reflexión y justificación, debidamente argumentada, sobre la naturaleza filosófica, pedagógica y epistemológica desde la cual se pensó y elaboró el diseño curricular. Más aún, se encontró que, incluso dentro de las limitaciones indicadas, el documento original de formulación del Plan de Estudios es mucho más rico en términos conceptuales y de explicación de la intencionalidad educativa, que el documento de reformulación, el cual desde nuestra perspectiva, se limitó a introducir ligeras variantes y a editar el texto original, pero sin clarificar ni actualizar la fundamentación curricular ni su contextualización a las realidades cambiantes de una sociedad que, al cabo de poco más de una década, había experimentado transformaciones decisivas en todos los órdenes.

Así pues, el análisis hermenéutico requirió un esfuerzo mayor para desentrañar los significados latentes en el corpus de textos estudiados. 
Gracias a las entrevistas en profundidad fue posible ahondar en el imaginario y las valoraciones de los actores consultados: coordinadores del posgrado, profesores con amplia trayectoria -y que incluso participaron en la redacción del plan original-, y autoridades de la División de Educología que egresaron de la Maestría en Educación, lo que contribuyó significativamente a enriquecer el ejercicio de interpretación del proceso de construcción cultural del currículo, que aquí se expone.

\section{Cartografiando rutas y sentidos: visiones y saberes en disputa en el Plan de Estudios}

El primer propósito de la investigación consistió en analizar el proceso de construcción cultural del currículo de la Maestría en Educación con énfasis en Docencia Universitaria, en el período 1998-2011, con especial interés en la identificación crítica de las racionalidades curriculares dominantes en su justificación y formulación filosófica, epistemológica y pedagógica. En consecuencia, el abordaje de la construcción cultural llevó a considerar, tanto en el análisis de contenido como en las entrevistas, las ideologías subyacentes en el Plan de Estudios y las concepciones en torno al sentido que se le otorga a la docencia en relación con la universidad y la sociedad.

En otras palabras, interesó rastrear las visiones de mundo que permean la propuesta académica del Plan de Estudios y las interpretaciones que hacen de este algunos de sus actores, lo que constituiría, siguiendo a Apple (2008), una prueba de la presencia de la hegemonía ${ }^{2}$ en el campo curricular, por la vía de las tradiciones selectivas y legitimadoras del conocimiento, y de todas aquellas presuposiciones ideológicas que, de forma más o menos consciente, "proporcionan las reglas lógicas para el pensamiento y la acción de los educadores" (pp. 17-18).

Un hecho que consideramos relevante, y que se inscribe en la tesis que entiende al campo curricular como un escenario o espacio de disputa de fuerzas ideológicas y de intereses de distinta naturaleza, es

2 Raymond Williams (citado en Apple, 2008, p. 16) define la hegemonía como un conjunto total de prácticas y expectativas que moldean el entendimiento del hombre sobre su situación particular, su relación con el colectivo y sobre el mundo: "es una serie de significados y valores que, en la medida en que son experimentados como prácticas, aparecen como recíprocamente confirmantes. Constituye así un sentido de la realidad (...), un sentido de lo absoluto porque se experimenta como una realidad", fuera de lo cual los miembros de un grupo o comunidad experimentan dificultades "para moverse en la mayoría de las áreas de su vida". 
la reformulación de la intencionalidad formativa del plan de estudios, visible al comparar los documentos de 1998 y de 2009. En el primero, se define como objetivo fundamental del programa de Docencia Universitaria, en su conjunto, "la formación pedagógica en el campo de la Educación Superior, que permita a estos profesionales enfrentar con éxito sus funciones" (MED, 1998, p. 2). En la conversación con uno de los profesionales que redactó este texto, quedó en evidencia la preocupación que tuvo el equipo de trabajo por imprimirle a la propuesta de formación un sello de clara orientación pedagógica, que superara las visiones estrechas de las escuelas y facultades de la UNA que "querían recetas para sus profesores":

Desde el principio, planteamos la necesidad de que el docente tuviera una formación no solo en métodos y técnicas de enseñanza, que inclusive era la exigencia de las facultades [hacia el CIDE] (...) De ahí nuestro interés porque la formación en docencia universitaria trascendiera lo que era solamente la enseñanza, y que el plan de estudios diera cuenta también de cursos que fortalecieran las destrezas y competencias para la elaboración de un plan de estudios, para el diseño curricular... es decir, para que tuvieran un bagaje más amplio. (...) Que la puerta de entrada al quehacer docente no fuera únicamente la profesión, sino la formación pedagógica. (C. Lépiz, comunicación personal, 8 de octubre, 2013)

No obstante, como resultado del paso por las distintas instancias de aprobación a las que usualmente son sometidos los planes de estudio en la UNA, la mención en Docencia Universitaria no logró consolidar su apuesta por la pedagogía, y en su lugar se inclinó por el enfoque de las ciencias de la educación, con las implicaciones paradigmáticas que esto conllevaba. Como lo recuerda uno de sus impulsores:

Nosotros queríamos rescatar, a través de la Maestría, lo que había sido el programa de perfeccionamiento en docencia universitaria del CIDE (...). Pero en el proceso, como usted sabe, la propuesta tuvo que ir a muchas instancias: unidades académicas, consejo de CIDE, Vicerrectoría Académica, a los equipos curriculares de la UNA... y en todo ese itinerario se fueron dando discusiones, propuestas y contrapropuestas. Al final, lo que se vio era que 
resultaba más conveniente abrirlo y no restringirlo solamente a la pedagogía universitaria, para ampliar el campo de estudio y el perfil de entrada de los candidatos. [La inclinación hacia las ciencias de la educación] fue algo que surgió casi por conveniencia de hacia dónde debía orientarse el programa de Maestría y el plan de estudios a efecto de que tuviera una mayor aceptación dentro y fuera de la universidad. Por supuesto, hubiera sido muy enriquecedor para el CIDE y el posgrado tener un debate sobre paradigmas en la formación docente. Pero eso no se dio. (C. Lépiz, comunicación personal, 8 de octubre, 2013)

Destacamos esta cita, a pesar de su extensión, porque ilustra bien la dinámica de construcción cultural del currículo que se propuso investigar: ese proceso complejo de creación de propuestas, de discusión y de disputas en el campo curricular, pero también, en el político y el ideológico. En este caso, además, se evidencia una tensión entre los paradigmas e ideas del equipo que diseñó la propuesta de mención en Docencia Universitaria, y lo que podríamos llamar la razón institucional universitaria, amparada probablemente en criterios de rentabilidad económica (toda vez que los posgrados deben buscar su autosostenibilidad financiera) y de conveniencia; no obstante, también podemos ver aquí la acción de las normas "constitutivas o subyacentes [que] exigen que se enseñen currículos centrados en temas, [en los] que se conceda un alto estatus al conocimiento técnico" (Apple, 2008, p. 57) -el conocimiento legitimado de las ciencias de la educación-, cuya lógica de selectividad de los saberes funciona como dispositivo o filtro cultural de lo que debe enseñarse y aprenderse, y del lugar epistemológico desde el cual se deben realizar tales operaciones.

Dicha tensión se va a resolver -aunque no necesariamente para bien- en el documento de rediseño del año 2009, cuando la perspectiva pedagógica desaparece formalmente como orientación curricular; en su lugar, se consolida una visión del posgrado en la que se concibe como una propuesta de formación cuyo sentido se encuentra solamente en la posibilidad de "satisfacer las necesidades de formación y capacitación de recursos humanos, en la temática acerca de los procesos institucionales en la Educación Superior" (MED, 1998, p. 3; Villalobos, 2009, pp. 6-7), como también lo establecía el Plan original, pero ahora sin el peso que tenía antes la formación pedagógica. Dicho cambio se intenta 
justificar en virtud de la exigencia de "profesionalización de la tarea educativa" que demanda la época actual, y cuya legitimidad estaría dada por una suerte de herencia derivada de la historia de la universidad y de la misión del CIDE de "formar, capacitar y actualizar a los académicos de la UNA, para elevar cualitativamente su formación docente" (Villalobos, 2009, p. 8).

Esta es, pues, la finalidad del Plan de Estudios actual, a partir de lo que se configura una visión instrumental de la docencia universitaria -y del ser docente y su rol en la sociedad-, basado en la adquisición de unas ciertas prácticas didácticas y la apropiación de una selección de saberes técnicos. No es un dato menor que en el Plan de Estudios vigente se entienda la "labor docente" como "indispensable para asegurar la consistencia entre los resultados y los logros previstos en el currículo, en el contexto de las necesidades, aspiraciones y exigencias de la sociedad contemporánea" (MED, 1998, p. 4; Villalobos, 2009, p.7. El resaltado no corresponde al original); ni tampoco, el que al avanzar hacia los objetivos del Plan se encuentren, como brújulas del proceso de formación, dos aspiraciones altamente iluminadoras de la imagen del docente universitario que subyace en la fundamentación del diseño curricular: una es la idea de "formar personal para orientar, planificar y ejecutar procesos de enseñanza y aprendizaje en la Educación Superior"; y otra, la de fomentar "la necesidad de perfeccionamiento en el cumplimiento de la labor docente de una institución" educativa (Villalobos, 2009, p.7).

Aquí, tanto el trasfondo ideológico y cultural, como el lenguaje en que se expresa la voluntad y la intencionalidad formativa del Plan de Estudios, revelan una concepción de la docencia universitaria y del educador permeada fuertemente por su inserción institucional, por su capacidad adaptativa a las condiciones y requerimientos de ese medio, y por su protagonismo (más que el del estudiante) en la consecución de los objetivos y logros educativos; en contraposición, el desarrollo de su pensamiento crítico, su capacidad reflexiva y problematizadora de la educación desde el dominio filosófico, pedagógico e incluso epistemológico de los saberes y las prácticas docentes, no se postulan como propósitos explícitos.

Paradójicamente, en el documento del año 2009, que propicia esta invisibilización de la pedagogía, tal cambio se justifica con el argumento de la responsabilidad histórica de la UNA, como "heredera 
del pensamiento educativo que formó a las y los docentes de este país, desde los albores del siglo pasado, cuando se fundó la Escuela Normal de Costa Rica" (Villalobos, 2009, p. 7), cuando lo cierto es que una exploración de los referentes intelectuales y los principios que constituyen ese legado que se invoca, no podrían llevarnos a otro lugar que no sea el de la pedagogía. Este recorte de lo pedagógico, y el posicionamiento, en su lugar, del enfoque de las ciencias de la educación, expresa una tendencia clave en este proceso de construcción cultural del currículo, toda vez que da pie a una suerte de invisibilización de la pedagogía como eje vertebrador del Plan de Estudios, con consecuencias sobre el desarrollo curricular y sobre las concepciones que docentes y administradores curriculares van a ir construyendo y reelaborando a lo largo del tiempo, desde sus propias experiencias y paradigmas.

En efecto, a pesar de sus diferentes puntos de vista, los informantes consultados coinciden en identificar ese giro paradigmático de la pedagogía a las ciencias de la educación, y el énfasis en lo instrumental por sobre lo reflexivo/crítico, como uno de los aspectos claves para comprender la evolución del Plan de Estudios de la Maestría en el período que aquí estudiamos. De los diálogos sostenidos al respecto, se pueden identificar tres grandes enfoques interpretativos.

El primero sugiere que la preeminencia de los saberes de tipo práctico y procedimental "de alguna manera empujan a aplicar estrategias, más que a reflexionar sobre esas estrategias", lo que obedecería a dos causas: una coyuntural, relacionada con el contexto en el cual se diseñó y aprobó el Plan de Estudios (el período de los años 1990), "muy cargado de ideas y tesis sobre la necesidad de formar mano de obra, de hacerlo rápido y de ponerla a trabajar para producir dinero", y cuya impronta no ha sido corregida hasta hoy; y la otra causa, propia de la cultura organizacional -y acaso curricular- del posgrado, que acusa la superficialidad del rediseño del año 2009, puesto que en esa ocasión "se tomó lo que ya estaba establecido y planificado, para tratar de mejorar vacíos y replantear cosas", pero no se propició "un proceso de reflexión fuerte sobre qué es lo que se quiere y qué es lo que se necesita actualmente" en materia de docencia universitaria (S. Jiménez, comunicación personal, 10 de abril de 2013).

El segundo enfoque comparte la crítica a la superficialidad del rediseño del Plan de Estudios, además, señala que el peso excesivo de las ciencias de la educación fragmenta el saber y añade dificultades 
para el proceso de diálogo entre "las disciplinas pedagógicas". En este caso, la contradicción se daría con respecto del objeto de estudio -la docencia universitaria- que sí remite "a un posicionamiento más pedagógico", donde la psicología de la educación, la filosofía de la educación, el currículo, la didáctica y otras disciplinas más, "confluyen para resolver problemas propios de la docencia universitaria" (G. Sánchez, comunicación personal, 19 de setiembre de 2013). Que se siga hablando de ciencias de la educación en nuestros días, consideró uno de los entrevistados, "limita mucho la formación de los estudiantes" y tiene implicaciones en la construcción de las concepciones "sobre lo que es la enseñanza y el aprendizaje, y eso obviamente tendrá repercusiones en la forma en que van a diseñar su mediación pedagógica" (G. Sánchez, comunicación personal, 19 de setiembre de 2013).

Por último, el tercer enfoque explicativo va más allá en la interpretación del giro paradigmático hacia las ciencias de la educación que experimentó la Maestría y su Plan de Estudios, y propone que se trató de una decisión "consciente e intencionada", con la que si bien no se renuncia "a la pedagogía como eje de la formación docente", sí se pone de manifiesto "la primacía del conocimiento (...) y de la ontología" por sobre el saber ético que sería el propio de la docencia. En esta línea argumentativa, uno de los entrevistados afirmó que la inclinación por las ciencias de la educación, y hacia una visión más instrumental de la docencia, obedece al triunfo de "razones históricas-institucionales y consignas teóricas-metodológicas" en el CIDE: "en el claustro académico no se ha podido establecer de manera explícita e inequívoca de manera oficial, o al menos de manera tácita, lo que debería constituir la pedagogía que se propone para el ejercicio profesional de la docencia universitaria" (C. Méndez, comunicación personal, 10 de octubre de 2013).

Estos criterios confirman algunas de las premisas y características diagnosticadas en la literatura especializada, en torno a los procesos de construcción del currículo y la formación docente en América Latina. Los problemas que se observan a nivel regional, por ejemplo, las estructuras curriculares casi invariables, basadas en conocimientos científico-disciplinares más que pedagógicos; el peso de la administración burocrática sobre la gestión formativa; "el predominio de la lógica técnico instrumental en los procesos de elaboración de los currículos"; la inviabilidad del trabajo interdisciplinario o "el énfasis en la medición de productos-resultados de la formación" (Pinto, 2008, pp. 
56-57), afloran también al hurgar en el tejido curricular de la Maestría en Educación.

El currículo como campo cultural se nos revela, así, en toda su conflictividad y complejidad: inmerso en tensiones por el control del conocimiento y de la sociedad, o bien, impulsando las rupturas emancipatorias en medio de no pocas resistencias, en línea con aquellas tesis que ven más allá de la reproducción ideológica y que, por el contrario, conciben el currículo como posibilidad creativa de liberación del ser humano y de la comunidad educativa.

\section{Elementos dominantes de la racionalidad curricular del Plan de Estudios}

Las visiones expuestas y analizadas en el apartado anterior nos introducen al ámbito de las racionalidades curriculares, las que constituyen el prisma -o mediación sociocultural- desde el cual los actores que participan del diseño y ejecución del currículo articulan sus concepciones ideológicas y sus "opciones de poder, de saber y de hacer", y que, por su fuerza reguladora y ordenadora del mundo referencial del sujeto y de los procesos educativos, se convierten en "acciones históricas que se expresan en el lenguaje, y en cuanto tales, orientaciones determinantes para el saber y el hacer" (Pinto, 2008, pp. 92-93).

¿Cómo se podrían caracterizar los elementos dominantes de la racionalidad curricular que dan sentido a la Maestría en Docencia Universitaria? A partir del análisis de la justificación y la fundamentación del Plan de Estudios, fue posible identificar las relaciones que se establecen entre la concepciones sobre la docencia y los ejes curriculares de la formación docente, que se expresan en los documentos de los años 1998 y 2009, y que constituyen regulaciones para el desarrollo del currículo -independientemente de que se asuman como tales o no por parte de los responsables de la gestión-.

Una vez más, al realizar un ejercicio comparativo, se advierte un mayor desarrollo teórico y argumentativo en el Plan de 1998, especialmente, en lo que hace a la conceptualización de "las habilidades didácticas del educador y la organización de la enseñanza", que giran en torno a cuatro aspectos básicos: la utilización adecuada del proceso de la comunicación ("eje del proceso de enseñanza-aprendizaje"); la aplicación de diversos enfoques, métodos, técnicas y recursos de 
enseñanza (cuya "eficacia" dependerá "de la situación, la madurez de los alumnos, los objetivos que se deben lograr"); la evaluación del aprendizaje ("el docente debe elaborar instrumentos con referencia a criterios, si quiere medir logros y determinar procedimientos para mejorar sus habilidades") y la estimulación del pensamiento reflexivo ("es fundamental que los estudiantes desarrollen esta habilidad, porque el hombre, como ser social, permanentemente está enfrentando desafíos y nuevos problemas") (MED, 1998, p. 4-10).

Estos aspectos se omiten totalmente en el rediseño del 2009, aunque esa suerte de espíritu eficientista de la docencia -y de la educación, en general- permanece. Sobre esa exclusión del pensamiento reflexivo como eje del Plan de Estudios, una de las personas que se ha desempeñado como coordinador del posgrado sostuvo que, efectivamente, hacen falta los espacios de reflexión en la formación de docentes universitarios, pero no es un problema exclusivo del posgrado:

Si comparo con los programas de bachillerato y licenciatura, tampoco ahí hay tantos espacios para analizar críticamente qué significa ser docente y ser educador. Esta es una debilidad que presenta el Plan en sí mismo, pero que también arrastramos desde los niveles previos de formación. Es decir, un problema de la unidad académica y del CIDE. (C. Lépiz, comunicación personal, 8 de octubre, 2013)

Asimismo, en el documento del 2009 se reitera la noción de la Docencia Universitaria como un área académica compleja -que también estaba expuesta en 1998-, por lo que requeriría de un abordaje interdisciplinario en el que participan disciplinas como "la Psicología, la Educación, la Sociología, la Antropología, y la Andragogía, entre otras, cuyos aportes ayudan a comprender y situar los procesos de la Educación Superior en su contexto social e institucional" (Villalobos, 2009 , p. 8). Además, se propusieron cuatro ejes curriculares, a manera de grandes rutas de la acción educativa: la teoría de la educación, que "proporciona los conceptos fundamentales más generales de esta área del saber"; la investigación, que permitirá "la formación de un profesional con mentalidad abierta a la interrogación acerca del qué, el cómo y el porqué de los hechos"; la educación superior, "con el propósito de encontrar alternativas viables que mejoren el proceso académico, desde 
la perspectiva de los estudiantes, de los docentes, de los productos generados en la academia, y de los procesos para llegar a esos productos"; y el desarrollo académico, "cuyo objetivo es el logro de la excelencia en el quehacer académico" (Villalobos, 2009, pp. 9-10).

Llama la atención que en esta revisión y actualización del Plan de Estudios se eliminara uno de los ejes curriculares (ciencias aplicadas) y que, en su lugar, se incluyera otro denominado educación superior, homónimo de una de las áreas disciplinarias, y cuya descripción es una copia casi literal de uno de los párrafos del eje de desarrollo académico del Plan de Estudios de 1998. Más sorprendente aún es que las instancias de revisión de diseño curricular de la UNA no advirtieran este hecho, y que aprobaran sin más la nueva formulación. Finalmente, una modificación formal que se registra en este documento es la inclusión de "los ejes transversales propuestos por la Universidad Nacional (...) a saber, género, equidad, desarrollo sostenible, diversidad cultural, ambiente y cultura ambiental" (Villalobos, 2009, p. 8). Sin embargo, estos ejes no se reflejan en la malla curricular ni en los contenidos temáticos de los cursos.

Es evidente que el enfoque de formación basado en las ciencias de la educación prácticamente pone sello de identidad al Plan de Estudios de la Maestría y, al menos en términos nominales, enmarca su andamiaje curricular, al punto de que, más allá de algunas menciones esporádicas en los documentos, la pedagogía ni siquiera es considerada como un eje curricular.

A pesar de que históricamente esta opción paradigmática terminó por convertirse en hegemónica, parece que no ha logrado influir en la construcción teórica y práctica del objeto de estudio del posgrado, a saber, la docencia universitaria. Esta es una conclusión que emerge de las reflexiones de los entrevistados, y que se plantea en dos planos: uno de ellos alude a un dilema sustancial del Plan, es decir, la tensión existente entre la pedagogía y las ciencias de la educación como enfoques teóricos de la formación docente. Si bien es la última la que se ha impuesto, en términos curriculares esto respondería más a la necesidad de justificar la incorporación de "cursos dentro de una línea curricular", pero no logra responder al porqué de tal elección. Al respecto, dice una de las informantes: 
[El plan de estudios] no dice qué son ciencias de la educación y qué es pedagogía, tampoco dice si coexisten, o tan siquiera dice si considera a la pedagogía como una de las ciencias de la educación. Es decir, dentro de todas las posibilidades que podríamos tener, no dice claramente cuál es el posicionamiento teórico que asume el Plan de Estudios. (S. Jiménez, comunicación personal, 10 de abril de 2013)

El otro plano incorpora al debate la mirada ética de la docencia universitaria: una dimensión que no ha sido considerada hasta ahora en el diseño curricular ni en la construcción de significados en torno a este. Al respecto, uno de los entrevistados, con amplia experiencia académica en la UNA y como docente del posgrado, plantea la necesidad de que, en el marco de los procesos de discusión sobre un posible nuevo Plan de Estudios, se aborde el de la docencia como un problema ético antes que epistemológico y "ni tan siquiera ontológico", es decir, "como una situación en la que la alteridad, el otro, es inclusive más importante que uno mismo" (C. Méndez, comunicación personal, 10 de octubre de 2013).

La cuestión de fondo, entonces, es otorgar un lugar de mayor preponderancia al humanismo en la docencia universitaria, como señal de identidad de los procesos educativos en una universidad como la UNA que, en principio, desde sus valores fundacionales y sus herencias pedagógicas, responde a una visión de universidad necesaria, mucho más comprometida con el bienestar y el desarrollo pleno del ser humano y de la sociedad. Así, lo que se señalaría es una ausencia o un vacío en la fundamentación del Plan de Estudios de la Maestría, y en concreto, de su objeto de estudio, que aún no ha sido subsanado ni puesto en discusión abierta para delinear nuevas cartografías de la formación de docentes universitarios. Algo que la racionalidad curricular dominante no estaría permitiendo, al menos en lo que se plasma en un texto o documento oficial. En ese sentido, dice la persona entrevistada:

(...) la perspectiva de la docencia se ha visto como una prolongación o como un quehacer administrativo, por así decirlo, como una obligación, pero no tiene todavía ese sustento teórico de la antropología, o de la filosofía [de la educación], que nos diga con claridad que la docencia es otra cosa, que no es la aplicación de la 
investigación, sino que es la interacción entre personas, el respeto por el otro, el reconocimiento de su unicidad como persona. $\mathrm{Y}$ esto no por el simple hecho de ser un ser humano, sino de ser un docente, y de manera particular en esta universidad (...) Por eso decía que para mí es suficiente lo que dice el actual plan de estudios, pero no estoy de acuerdo. Está bien escrito, orienta, pero ese mapa no lo quiero porque no me gusta andar por ese territorio. Yo necesito otras rutas. (C. Méndez, comunicación personal, 10 de octubre de 2013)

¿Hacia dónde conduce el mapa actual del Plan de Estudios? La hegemonía de la visión instrumental orienta a la Maestría, y a su énfasis en Docencia Universitaria, hacia una zona curricular muy próxima a la racionalidad adaptativa (Pinto, 2008), reproductora del statu quo dominante en la comunidad educativa.

\section{Principios de incertidumbre...}

$\mathrm{Si}$, en términos de las racionalidades curriculares, uno de los principales hallazgos de la investigación fue el de la existencia de una cartografía de la formación en docencia universitaria que conduce a rumbos poco claros o no deseables para los actores del currículo (como el de la racionalidad adaptativa); desde el punto de vista de los principios filosóficos el aspecto más destacado es la contradicción que parece estar instalada en el Plan de Estudios: por una parte, y al menos con carácter declarativo, se afirma que el programa de Maestría es congruente "con los principios humanistas de libertad, autonomía, respeto, todo lo cual se dirige al logro del desarrollo humano, tanto en el plano individual, como en el social" (Villalobos, 2009, p. 10), lo que sugiere un abordaje amplio, progresista y con un enfoque que privilegia claramente la dimensión humana de la formación docente y de la educación, como un todo. Pero, por otra parte, el análisis evidenció una visión instrumental de la docencia que recorre transversalmente los documentos curriculares. Inclusive, al consultársele sobre el tema a una de las personas entrevistadas, esta reconoció que "es difícil decir cuáles son los principios o fundamentos filosóficos (...) no está claro cuál es el posicionamiento que tiene el Plan de Estudios" (S. Jiménez, comunicación personal, 10 de abril de 2013) en este tema. 
En cuanto a los principios pedagógicos, estos tampoco aparecen explícitamente en el documento, más allá de algunas menciones aisladas en distintos apartados de la fundamentación. ¿Qué implicaciones tendría este vacío o ausencia en el Plan de Estudios? Como ya se indicó antes, el hecho mayúsculo de la ausencia de un componente de pedagogía como área disciplinar o como eje curricular de la formación de docentes universitarios, tiene consecuencias potencialmente negativas: acaso la principal sea el riesgo de reducir la enseñanza a una simple transmisión de conocimientos, a un adiestramiento en prácticas específicas, generando con esto aprendizajes que no son necesariamente significativos y que no transforman las prácticas educativas, toda vez que estarían desprovistos de fuertes anclajes teóricos, epistemológicos y hasta políticos y culturales.

En sintonía con esto, uno de los informantes afirmó que la fundamentación filosófica y pedagógica del Plan de Estudios podría ser descrita "a partir de adjetivaciones como las siguientes: contenido suficiente pero lacónico y más bien superficial y correcto, pero un poco difuso a veces. En lo sustantivo, la fundamentación me resulta desalentadora"; y aunque reconoció que esta valoración puede resultar injusta, la defiende por cuanto "no me complace el paradigma que conlleva o asume esa fundamentación (que, por otro lado, es o constituye el caballo de batalla argumental más frecuente en muchos recintos, predios o campus)" (C. Méndez, comunicación personal, 10 de octubre de 2013). Un paradigma que no es otro sino el paradigma positivista de las ciencias de la educación.

En lo que el Plan de Estudios sí establece una mejor caracterización es respecto del modelo pedagógico de la mención en Docencia Universitaria, al que define como "un modelo interactivo de aprendizaje y comunicación", basado en un proceso de "intercambio de conocimientos entre las y los estudiantes y profesor (as), mediante una comunicación eficaz y eficiente, la cual se favorece mediante la construcción de ambientes de aprendizaje de tipo igualitario, democrático, participativo, crítico y creativo" (MED, 1998, p.6 ; Villalobos, 2009, p. 10).

Otro componente de este modelo, al menos en su descripción, es el que pretende "establecer la realidad como punto de partida" del proceso de aprendizaje, con miras a aprovechar "las experiencias prácticas de los participantes como materia prima para la discusión, el estudio de casos, y la aplicación de otras técnicas y procedimientos de análisis"; 
esta intervención sobre la realidad se propone a partir de la observación y análisis de lecciones, entrevistas, cuestionarios, y otras técnicas "aplicables a la indagación en situaciones de aula" (MED, 1998, p.6 ; Villalobos, 2009, pp. 10-11).

Como se puede apreciar, el modelo pedagógico que se enuncia está centrado - a nivel de la propuesta- básicamente en las relaciones horizontales entre docentes y estudiantes, y en la idea de que el aprendizaje se logra a partir de la práctica, la investigación y la comunicación (puesta en común) de los conocimientos y de las experiencias docentes. No obstante, tampoco aquí se observa un posicionamiento teórico y axiológico consistente para orientar tales procesos, acaso como consecuencia de esas contradicciones señaladas antes, en términos de la ambigüedad -y acaso superficialidad- de sus principios filosóficos, pedagógicos y epistemológicos.

En un acercamiento inductivo, y partiendo de la noción de modelo pedagógico de Florez (1994), y de su tipología, en el Plan de Estudios del énfasis en Docencia Universitaria sería posible identificar algunos rasgos propios de los llamados modelos cognitivos, especialmente, de la tendencia conocida como de la enseñanza basada en el descubrimiento, es decir, aquella que "privilegia los conceptos y las estructuras básicas de las ciencias", y en la que "los alumnos realizan su aprendizaje a medida que experimentan y consultan la bibliografía disponible, analizan la información nueva con la lógica del método científico y deducen sus propios conocimientos" (Florez, 1994, p. 189).

Sin embargo, al confrontar nuestra perspectiva con la de las personas entrevistadas, comprobamos que tampoco para ellas existe ni la claridad ni el consenso sobre el modelo pedagógico que, desde sus distintos roles como gestores curriculares, han venido desarrollando. Así, por ejemplo, uno de nuestros informantes reflexionaba en estos términos:

No tenemos un modelo pedagógico tradicional, pero tampoco somos críticos; podríamos estar cerca del modelo de ciencias cognitivas. Dentro de ese gran bloque de constructivismo, que va de lo más tradicional a lo más crítico, nosotros estamos al centro, en un punto medio pero muy desde el punto de vista cognitivo, y no de transformar la realidad. Es decir: entiendo, interpreto y posiciono a la persona para que él mismo construya su conocimiento en la parte de educación y docencia, pero tampoco estamos exigiendo 
o pensando que debe transformar esa realidad educativa en la que está inmerso. Por lo menos no lo dice abiertamente el Plan de Estudios, y yo tampoco lo veo (S. Jiménez, comunicación personal, 10 de abril de 2013).

Otro de los entrevistados fue más lejos y ubicó el modelo pedagógico "dentro de los más arcaicos", "convencionales" y "rígidos" en materia de formación de docentes, y específicamente, lo define como preconstructivo, puesto que se concibió y diseñó entre, 1996 y 1997, "bajo unos criterios concretos y particulares, que respondían a la visión de unas personas, y en un contexto universitario muy concreto" (C. Lépiz, comunicación personal, 8 de octubre, 2013).

En contraste con las anteriores definiciones, un tercer juicio sobre el modelo pedagógico que articula la mención en Docencia Universitaria lo aproxima "por lo menos a partir del discurso que se propone como fundamentación (no necesariamente a partir de los programas de curso propios del plan de estudios)", como crítico constructivista (C. Méndez, comunicación personal, 10 de octubre de 2013).

Esta conjunción de factores y visiones en conflicto sobre los principios y modelos que sostienen la fundamentación curricular del Plan de Estudios afectan, a su vez, la consistencia teórica y metodológica de los procesos de formación en la práctica. Más aún, los criterios expuestos delinean un choque entre el paradigma positivista actual -enfoque de ciencias de la educación- y un paradigma emancipador -que definimos como humanista pedagógico- que emerge como deseable en la concepción y desarrollo de la docencia universitaria. Como lo sintetizó bien uno de los profesores que colaboraron en esta investigación, en el diseño y la fundamentación del Plan de Estudios:

(...) falta la parte teórica, el ideario, el mapa conceptual que nos diga qué es lo que estamos propiciando en el posgrado, qué es realmente docencia, [qué es] la enseñanza... ¿De qué está más cerca la docencia como campo de estudio: de la antropología filosófica, inclusive de la religión... iqué se yo!, antes que de un enfoque de tratamiento psicológico? ¿Por dónde andamos? Ahí es donde yo digo, y vuelvo a insistir, que el asunto es ético. (C. Méndez, comunicación personal, 10 de octubre de 2013) 
Si se parte de la tesis de que los principios filosóficos, pedagógicos y epistemológicos configuran, en más de un aspecto, la vida y el alma curricular de un plan de formación; y si, al mismo tiempo, se reconoce que su claridad y coherencia conceptual impregna las experiencias educativas y los procesos "de construcción de sujetos, de lenguajes, de acciones y de mundos" (Pinto, 2012, p. 60), entonces, en consecuencia, la Maestría en Educación tiene aquí un reto impostergable para redefinir su rumbo en medio de un cartografía curricular recorrida por contradicciones, incertidumbres y disputas de saberes y prácticas que, probablemente, hoy le impiden dar respuestas más oportunas y pertinentes a las exigencias actuales de la sociedad y de los sujetos que ingresan al posgrado.

\section{Ponernos a nosotros mismos como valiosos: pensar la docencia uni- versitaria desde y para América Latina}

El segundo propósito específico de la investigación consistió en evaluar, en forma cualitativa, las formas de abordaje o incorporación de la perspectiva pedagógica latinoamericana y caribeña, en el currículo de formación de docentes del programa de Maestría en Educación con énfasis en Docencia Universitaria. Para ello, se amplió el análisis de contenido de los documentos de formulación (1998) y rediseño (2009) del Plan de Estudios, para incluir una muestra de programas de curso de este mismo período, a los que se tuvo acceso en el archivo del posgrado. El punto de partida aquí fue un ejercicio intelectual de interpretación y búsqueda de respuestas, desde la teoría, a lo que, en la experiencia como estudiante de la Maestría, se había percibido como una problemática: a saber, la presencia marginal de América Latina en el diseño curricular y, de manera oscilante -de acuerdo con la mediación pedagógica de cada docente y sus referentes teórico-, en los procesos de enseñanza y aprendizaje que se propician en los cursos.

Como resultado de la lectura crítica de los documentos del corpus analizado, aquella presunción trocó en la confirmación de un hecho: las referencias identificadas en torno al pensamiento y las perspectivas pedagógicas latinoamericanas, y en un sentido más amplio, a lo que significa ser docente en América Latina, fueron mínimas, cuando no inexistentes (apenas datos de contextualización oun listado bibliográfico). En efecto, más allá de una frase que se incluye en el documento de 1998 como justificación de índole administrativa, y que se repite íntegra en el 
rediseño del 2009: "actualmente, varias universidades latinoamericanas ofrecen programas, proyectos y acciones, (...) característicos del campo de la docencia universitaria" (MED, 1998, p. 3; Villalobos, 2009, p. 7), no se evidencian posicionamientos concretos sobre perspectivas latinoamericanas de la pedagogía y de la docencia universitaria, que podrían aportar nuevas fuentes paradigmáticas, epistemológicas y filosófico-pedagógicas al Plan de Estudios.

Ante la prueba de las evidencias, surgieron nuevas preguntas de investigación: ¿cómo explicar estos "olvidos" y "ausencias" en un plan de formación de docentes en América Latina? ¿Podría tener alguna relación con esos vacíos, el contexto en el que se ha desarrollado la Maestría, a saber, el de los años noventa y principios de siglo XXI, marcado por las reformas neoliberales,? ¿Son estas omisiones un rasgo que se presenta únicamente en este Plan de Estudios o señalan una tendencia a nivel del CIDE, de la UNA y, en general, de la educación superior costarricense?

$\mathrm{Al}$ ahondar sobre estas inquietudes con los entrevistados, se recabaron nuevos elementos de juicio, necesarios para la comprensión de este fenómeno. De tal suerte, quedó claro que la opción por las ciencias de la educación como dominio paradigmático también tiene implicaciones en el orden de las relaciones del saber/poder, toda vez que traza la polaridad entre un saber nuestro -el de la rica cultura pedagógica latinoamericana- y un saber ajeno, siendo este último el que el sentido común y la racionalidad curricular asume como legítimo. Uno de los informantes lo explicó en estos términos:

Las teorías contemporáneas que se ven en el Plan de Estudios son propias de contextos anglosajones, lo que hemos tratado es de ajustarlas; pero [también] existen teorías que surgen de Latinoamérica para Latinoamérica, y que no haya cursos que tengan que ver con eso es una cuestión de la cual estamos privando a los estudiantes (G. Sánchez, comunicación personal, 19 de setiembre de 2013).

Ese dilema entre lo propio y lo ajeno tiene un trasfondo ideológico difícil de ocultar, por cuanto la manera en que histórica y culturalmente hemos construido nuestros saberes, determina posiciones y actitudes (desde la sospecha epistémica hasta la escisión identitaria que redunda en una negación de lo que somos) frente a la interpelación de lo latinoamericano. Las declaraciones de uno de los colaboradores en el 
proyecto de investigación así lo atestiguan, pues si bien reconoce un lugar clave para la pedagogía y el pensamiento crítico en la formación de los futuros docentes universitarios, al mismo tiempo, considera que este giro no debería tener como fundamento epistémico el referente latinoamericano pues podría convertirse en un enfoque "reduccionista (somos hijos de Occidente: de Atenas y de Jerusalem, no sólo somos hijos de Nicarao y Vázquez de Coronado)", por lo que concluye que "tal perspectiva crítica latinoamericana, si se asume de manera excluyente, no tendría necesariamente que enriquecer el plan de estudios" (C. Méndez, comunicación personal, 10 de octubre de 2013).

Este argumento revela sesgos importantes -conscientes o inconscientes-, de corte eurocéntrico, que esclarecen el porqué el pensamiento latinoamericano y su perspectiva pedagógica tropiezan con obstáculos para su incorporación a la propuesta curricular del posgrado: la cultura y el saber producido fuera de nuestro contexto regional sigue ejerciendo un ascendente decisivo sobre aquello que consideramos como conocimiento legítimo, especialmente en la educación. Lo que dice nuestro informante en cuestión, retrata este fenómeno:

Pocas, muy pocas universidades latinoamericanas son posicionadas a nivel mundial en lugares destacados, y aquellas que destacan a nivel latinoamericano no estoy muy convencido de que asumen, al menos de manera predominante, esa perspectiva (por supuesto sin olvidar quién y con qué propósito se proponen esos "ranking"). (C. Méndez, comunicación personal, 10 de octubre de 2013)

Junto a esto, los hallazgos de las entrevistas develaron una dimensión de la cultura académica institucional que también explicaría los vacíos y carencias en materia de reconocimiento e inclusión efectiva de los aportes del pensamiento filosófico y pedagógico latinoamericano en el Plan de Estudios de la Maestría en Docencia Universitaria: se trataría de una tendencia a invisibilizar la pedagogía $-\mathrm{y}$, en concreto, la que ha surgido en nuestra América- en la formación de docentes en la educación superior costarricense. "Casi que es lo políticamente correcto: quien no lo haga o, al menos, no manifieste que lo hace, sería en términos figurados un caso raro" (C. Méndez, comunicación personal, 10 de octubre de 2013), afirmó uno de los entrevistados. 
Para otro informante, también se trataría de una suerte de desconocimiento teórico a nivel del CIDE y, por supuesto, de la División de Educología, de las tendencias y corrientes pedagógicas latinoamericanas, situación que habría sido muy notoria en la época de creación de la Maestría ("había muy poca gente hablando sobre el tema en Costa Rica e investigando sobre esto"). En lo que se advierte como un criterio discutible en más de un sentido, pero, al fin y al cabo, válido en el afán investigativo, dicha situación obedecería al:

(...) perfil del docente del Centro [que] es un perfil de docente de aula, no son docentes investigadores y eso influye en el bagaje cultural sobre teorías pedagógicas; están trabajando más en la didáctica de sus disciplinas. Esa cultura institucional que se ha desarrollado, de profesores docentes [sic], tiene repercusiones en el diseño curricular y en la práctica de aula. (G. Sánchez, comunicación personal, 19 de setiembre de 2013)

Desde la perspectiva teórica construida para esta investigación, y en términos de ensayar interpretaciones sobre estas ausencias y omisiones, que eventualmente puedan ser consideradas en la formulación de un nuevo Plan de Estudios, es claro que la opción por incorporar una epistemología del Sur se presenta como una alternativa posible necesaria. Para ello, se presenta una reflexión articulada a partir de dos enfoques que se nutren de la sociología y la filosofia: la teoría de la producción de las ausencias de Sousa Santos (2006), y los aportes críticos del argentino Roig (2009) en torno a la constitución del pensamiento latinoamericano.

El primer enfoque plantea que la racionalidad Occidental moderna, positivista e instrumental -de la que, de alguna manera, es tributario el currículo de la Maestría en cuestión-, encubre y oculta la diversidad y las diferencias para excluirlas y, en el peor de los casos, aniquilarlas por la vía del epistemicidio (la negación de toda racionalidad otra). El intelectual portugués sostiene que "mucho de lo que no existe en nuestra sociedad es producido activamente como no existente, y por eso la trampa mayor para nosotros es reducir la realidad a lo que existe" (Sousa, 2006, p. 17). Algo que, en el caso de estudio, remite a lo que existe como regulación o prescripción curricular en un Plan de Estudios, donde también puede instalarse como hegemónica esa "racionalidad 
perezosa, que realmente produce como ausente mucha realidad que podría estar presente" (Sousa, 2006, p. 23).

El enfoque de Roig, por su parte, ubica la cuestión en el lugar filosófico necesario para emprender la tarea intelectual de todo pensamiento propio, y en particular, del latinoamericano: "ponernos a nosotros mismos como valiosos" (Roig, 2009, p. 81), como sujetos de y en la historia, como protagonistas, frente a la estrategia o ejercicio del "olvido" que ha estado implícito en la construcción de las identidades nacionales, así como de las identidades políticas y culturales hegemónicas en nuestra América, y que, por lo tanto, también se instaló como fuerza ideológica dominante en el sistema educativo.

Para Roig (2009), el olvido es expresión permanente, y que se actualiza con regularidad, de aquella vieja dicotomía entre civilización y barbarie, característica del pensamiento dominante -pero subordinado a la cultura metropolitana- de las élites intelectuales y políticas latinoamericanas:

Se hace necesario rever la contradicción entre "civilización" y "barbarie", que atraviesa íntegramente el discurso político del siglo XIX y que, a su modo, pervive en nuestros días. La "civilización" exigía un olvido de la "barbarie" (...) un problema de determinadas formas culturales, en particular, hábitos, costumbres, modos de vida, de los que era necesario desprenderse para poder ingresar en el orbe cultural [hegemónico]”. (Roig, 2009, p. 69)

¿Acaso no sigue presente esa contradicción en el campo curricular, en la tradición selectiva del conocimiento que se enseña (legitimado) y del que se omite (el ausente, el olvidado); o en la definición de los referentes teóricos, filosóficos y epistemológicos que sustentan los diseños curriculares de un plan de estudios, e inclusive, de un posgrado como programa de formación de docentes? ¿Hasta qué punto somos conscientes de esta dinámica de inclusión/exclusión de los saberes, y de sus implicaciones en términos de reproducción de la hegemonía en la formación de formadores a nivel universitario?

Roig (2009) planteaba una tesis que puede orientar esta búsqueda de respuestas -desde lo propio- para comprender la naturaleza de los procesos educativos que hoy tienen lugar en las universidades, especialmente las públicas: 
El punto de partida para una definición de lo "nuestro" y del "nosotros" ha de ser siempre el sujeto concreto inserto en su mundo de relaciones humanas, desde el cual recibe o se apropia de formas culturales, y no lo recibido en sí mismo, cuya riqueza intrínseca se juega toda entera en el acto de recepción. Se hace por tanto necesario estudiar ese acto dentro del vasto proceso de incorporación de nuestra América a lo que se ha dado en llamar el Occidente. (p. 63)

A partir de la comprensión de ese dilema implícito en la historia y cultura latinoamericanas, y desde el doble posicionamiento teórico al que se hizo referencia, es posible develar la producción de las ausencias y los olvidos en un ámbito tan delicado como el del currículo y la docencia universitaria, a la vez, desmontar su andamiajes ideológicos: la monocultura del saber y del rigor (que presupone que solo el conocimiento científico es legítimo), la monocultura del tiempo lineal (funcional al Norte dominante y desarrollado, al que siempre tendremos que alcanzar), la monocultura de la naturalización de las diferencias (que al asumir como natural lo homogéneo, descalifica toda alternativa a lo hegemónico), la monocultura de la escala dominante (que nos somete al universalismo de la globalización, y con esto, "la realidad particular y local no tiene dignidad como alternativa creíble a una realidad global"), y finalmente, la monocultura del productivismo capitalista, es decir, la idea de que el crecimiento económico y la lógica del mercado determinan el valor de la naturaleza, del trabajo humano y de la educación misma (Sousa, 2006, pp. 23-25).

Ahora bien, ¿por qué volver a las raíces del pensamiento pedagógico latinoamericano hoy, en el siglo XXI, en un mundo deslumbrado por el vértigo de la inmediatez y desprovisto de anclas con su pasado? ¿Será acaso un exceso de romanticismo latinoamericanista o, por el contrario, se trata de un imperativo que se desprende de las grandes tensiones culturales que recorren a nuestra región?

Más allá de estas especulaciones, lo cierto es que el legado intelectual, ético y pedagógico de muchos hombres y mujeres de la educación y la filosofía latinoamericana, constituye una fuente indispensable de conocimientos originales y creativos que nos permitirían a todos comprender a cabalidad el rol del docente en la escuela -como institución social- del presente y del futuro. Acaso también sea una puerta 
de oportunidad para revertir la lógica de la producción de las ausencias y de los olvidos que, históricamente, han limitado las posibilidades de construcción de nuestro ser como sujetos latinoamericanos, y así, han conspirado en la tarea opuesta: la de nuestra producción subordinada como objetos de apropiación y colonización material y simbólica.

Cuando Pinto (2012, p. 15) afirma que en América Latina necesitamos legitimar una nueva mirada sobre los principios filosóficos y pedagógicos de las prácticas formativas, desde paradigmas que consideren "la historia, las identidades socio-culturales, las tradiciones ético-políticas y pedagógicas que nos constituyen como territorios latinoamericanos", en realidad, lo que se está reclamando es una restitución del lugar protagónico de nuestros saberes. ¿Cómo avanzar en ese rescate de lo propio, sustento de todo proceso de construcción de nuevas realidades educativas en nuestros países? Una tarea prioritaria para dar pasos en la dirección sugerida por este autor es la de retomar la rica tradición del pensamiento latinoamericano, para contextualizar desde allí las tareas prioritarias y las dimensiones de la función docente en la actualidad. Es decir, se trata de abocarnos individual y colectivamente a la tarea de rastrear, y de identificar, las principales ideas pedagógicas que han estado vinculadas a las luchas emancipatorias de nuestros pueblos - pero también a sus fracasos-, a lo largo de ya varios siglos, para hacerlas presencia viva del currículo y de la práctica docente.

Más específicamente, en el proceso de diseño de un nuevo plan de estudios del énfasis en Docencia Universitaria, ese rescate de lo propio implicaría, primero, situarse en el paradigma curricular de la racionalidad crítico-transformativa (que se abre a la participación de los sujetos y comunidades, a la pluralidad y a la diversidad en su construcción), y no reproductora, o cuando menos adaptativa, como la que actualmente impera en el posgrado. Luego, un segundo momento de incorporación de lo latinoamericano comprendería la definición del horizonte filosófico, ético, pedagógico y cultural, desde el cual se concebirá al ser humano, a la educación y la docencia; así como una toma de posición respecto de la intencionalidad de la propuesta formativa, la cual entendemos que debe ser radicalmente distinta al actual enfoque curricular: esto es, se requiere una apuesta por un currículo de carácter emancipador, que pueda confrontar la visión que entiende y categoriza al docente como recurso humano. 
Por último, se requiere, de parte de la comunidad educativa que tendrá la responsabilidad de diseñar los aspectos operativos del currículo, y de ponerlo en práctica en el marco del posgrado, un convencimiento pleno sobre la importancia de conocer en profundidad las problemáticas sociohistóricas, culturales y, por supuesto, educativas de nuestra América Latina, y reflexionar críticamente sobre ellas, con las herramientas que provee el pensamiento y la pedagogía latinoamericanas: es decir, asumir el a priori antropológico que planteaba Roig (2009), para ponernos a nosotros mismos como valiosos en todo aquello que acometemos desde el dominio intelectual, y por supuesto, desde el dominio profesional docente.

Ese convencimiento es el que podría imprimir un sello distinto al plan de formación de docentes universitarios, pero este no puede alcanzarse como resultado de una imposición vertical o de una directriz proveniente de alguna oficina de diseño curricular de la UNA, y ni siquiera, como producto iluminado del trabajo de uno o varios intelectuales de la pedagogía. La posibilidad de incorporar una perspectiva latinoamericanista en el plan de estudios pasa por asumir, como colectividad, un proceso de re-conocimiento, de autoafirmación de lo propio, y de ruptura creativa con la inercia curricular y con la racionalidad instrumental que hoy parecen ser dominantes en la cultura institucional del posgrado y de la universidad.

\section{A manera de cierre y para abrir nuevos debates: hacia un perfil del do- cente universitario en el actual contexto nacional y latinoamericano}

Con el último propósito específico de la investigación, se contrastó el perfil de salida del docente universitario que aspira a formar el actual currículo de la Maestría en Educación con énfasis en Docencia Universitaria, con respecto del perfil que delinea la propuesta de formación de docentes latinoamericanos situados (Pinto, 2012), construida desde el paradigma crítico transformador de la educación; esto, con miras a identificar saberes y prácticas emergentes para ser considerados en el diseño de un nuevo plan de estudios. Esta perspectiva de los docentes situados ha sido trabajada, en los últimos años, por el pedagogo chileno Rolando Pinto, a partir de sus experiencias como docente e investigador en numerosos países de América Latina. 
En los dos documentos que definen la propuesta de Plan de Estudios del posgrado, y a pesar de estar separados en el tiempo por más de una década, el perfil del egresado, es decir, la visión ideal de ese docente universitario que se está formando y profesionalizando, no varía más que en aspectos formales de presentación de la información; pero los aspectos de fondo, aquellos que son sustanciales y que expresarían una respuesta pertinente al diagnóstico previo de la realidad socioeconómica y educativa que hace posible el diseño curricular, están ausentes. De nuevo, la huella de la inercia institucional se descubre aquí como una traba al proceso educativo y a la administración misma del currículo que, por desactualizado, no puede responder efectivamente a las exigencias y expectativas que hoy genera entre las personas que ingresan a cursar la mención en Docencia Universitaria.

En un ejercicio de síntesis, elaborado a partir del análisis de los documentos de 1998 y 2009, el perfil del docente universitario que se postula desde el Plan de Estudios comprendería el dominio de competencias o atributos en tres campos básicos: saberes científicos sobre la educación, prácticas docentes y actitudes profesionales, todas articuladas en torno a un sentido de la profesionalización que, como se explicó en anteriores apartados, termina por expresarse como una visión instrumental, poco reflexiva y crítica del ser docente y de la docencia misma.

Al comparar este perfil con el que propone el enfoque de formación de docentes latinoamericanos situados, se encontró que, de cara a la revisión crítica de su diseño curricular y a su proyección en el futuro, la Maestría en Educación podría considerar nuevos elementos propios de la perspectiva nuestroamericana que enriquecerían, de manera sustancial y profunda, la propuesta formativa en aspectos tales como el protagonismo de los saberes pedagógicos (que se expresan en la organización del currículo, mediación pedagógica, decisiones didácticas, ética de la enseñanza, gestión de la formación) y el fortalecimiento de las competencias propias del ser docente (epistemológicas, pedagógicas, comunicaciones y transformativas de la práctica docente) (Pinto, 2012).

Estos saberes y competencias, a su vez, mantienen un hilo conductor en torno a las dimensiones culturales (multi e intercultural, en sentido amplio) del ser, del saber y del vivir en América Latina, y a la construcción de una razón emancipadora. Como lo explica Pinto (2008): 
Se trata que la educación se asuma como un desafío histórico-cultural de selección, organización y transmisión de la multiculturalidad [y la interculturalidad], esto es, de instalar en la escuela (...) recortes de espacio-tiempo en los que se puedan distinguir posibilidades de prácticas culturales concretas, que vayan permitiendo la construcción de sujetos latinoamericanos con visión de futuro, con identidad fundada en "lo propio y no en lo ajeno", en fin, una identidad fundada en una racionalidad democrática de lo diverso. (p. 212)

En general, la necesidad de poner en marcha un proceso de revisión de la pertinencia, la actualidad y la consistencia teórica y metodológica del Plan de Estudios fue reconocida por todas las personas entrevistadas en esta investigación, a quienes también se les consultó cuál sería, desde sus experiencias y perspectivas paradigmáticas, el perfil de formación de docentes hacia el cuál debería avanzar hoy el énfasis en Docencia Universitaria de la Maestría.

Los principales hallazgos de este ejercicio señalan una ruta de trabajo para las instancias de gestión académica del posgrado y de la División de Educología. En síntesis, se identificaron tres grandes áreas temáticas a partir de las cuales se podrían generar espacios de discusión y construcción curricular. En primera instancia, destaca la dimensión humanística de la docencia universitaria, "determinante en el aprendizaje" (C. Lépiz, comunicación personal, 8 de octubre, 2013), y que presupone un replanteamiento en profundidad del objeto de estudio de la Maestría (la docencia universitaria), a partir de aproximaciones filosóficas, pedagógicas, epistemológicas, éticas y estéticas. El propósito de este repensar el rumbo de la formación en cada uno de estos ámbitos, según el criterio de uno de los informantes, sería superar "ese fuerte arraigo o vocación" positivista de lo que se entiende por docencia universitaria en el CIDE, en particular, y en la UNA, en general, donde todavía predomina un abordaje "esencialmente epistemológico y ontológico", por encima del respeto y reconocimiento de la alteridad en el proceso educativo y en la mediación pedagógica:

El asunto debe ir por aquí: la docencia es un acto único, irrepetible, perece. Por eso si usted lo quiere analizar, debe registrarlo, porque si no desaparece. Entonces, la docencia es un arte, no una 
ciencia (...). Y por eso creo que la perspectiva axiológica -ética y estética- está totalmente ausente [del Plan de Estudios]. Si ese acto [la docencia] es bello, en el sentido estricto, lo es porque respeta esencialmente a la persona, es éticamente incuestionable, y eso es lo que yo quisiera que sea la docencia en el programa de maestría. Que sea el sello de identidad, lo que queremos hacer. No sé si venda o no; o si un profesor decide no usarlo, eso se respeta, pero nuestra propuesta es esa y busca abrir los ojos de los docentes en formación. (C. Méndez, comunicación personal, 10 de octubre de 2013)

Una segunda área temática es la de los saberes y prácticas docentes, en la que se involucran, por un lado, aquellos aspectos propios del saber (como conocimiento), del saber hacer y del saber vivir la docencia universitaria; y por otro lado, la integración de las tecnologías y las modalidades semipresenciales y virtuales de entrega de la docencia. Las opiniones de los colaboradores se mantienen muy cerca de la actual línea curricular del Plan de Estudios, lo que sugiere que, a pesar del paso del tiempo, la respuesta que se da desde el posgrado conserva todavía buena parte de su pertinencia. Aunque bien puede ser mejorada, por ejemplo, temas básicos de la docencia universitaria, como la mediación pedagógica, la didáctica, el currículo y la evaluación siguen siendo considerados prioritarios; $y$ se refuerza la importancia de trabajar en el estímulo de los saberes actitudinales y para la vida:

(...) el docente egresado debe ser una persona muy receptiva, investigadora de su propia realidad, con motivación para el aprendizaje constante en la enseñanza de su disciplina y la investigación de cómo enseñar en su disciplina; una persona que aprenda a leer muy bien las realidades de sus estudiantes, de los distintos contextos de los que provienen sus estudiantes, que sea sensible a la diversidad y atienda las necesidades de los educandos; veo a un profesor universitario que dialoga, y que no se limita simplemente a dar cátedra de manera dogmática, sino que identifique su contexto educativo y diseñe estrategias didácticas con un propósito pedagógico bien definido. (S. Jiménez, comunicación personal, 10 de abril de 2013) 
Asimismo, aparecen otros temas como la "gestión de proyectos de acción social y extensión, la atención de necesidades de educación no formal y de pedagogía social", que reclaman de la Maestría la audacia suficiente para integrarlos al Plan de Estudios (H. Ramírez, comunicación personal, 18 de octubre de 2013).

En este sentido, una idea importante, y acaso decisiva en la intención de transformar las concepciones y prácticas en torno a la docencia universitaria, es la de desarrollar en los estudiantes la capacidad de combinar saberes: "el saber pedagógico y didáctico, con el saber de su propia disciplina científica", para producir un nuevo "conocimiento pedagógico del saber disciplinario" (S. Jiménez, comunicación personal, 10 de abril de 2013). Lo mismo sucede con las nuevas tecnologías y las diversas modalidades en docencia que su aplicación pedagógica intencionada permite. Como reflexionaba uno de los entrevistados:

Con el advenimiento de las aplicaciones tecnológicas en la docencia, y con modalidades educativas como la virtualización o los programas semipresenciales, un docente ya no puede tener una formación para desempeñarse en una modalidad exclusivamente presencial. En estos momentos, sería totalmente desfasado que un docente no tenga destrezas para que, en cuanto se requiera, un curso se imparta de manera virtual; y esto implica todo un reto, por las resistencia que genera entre los mismos docentes. Se impone que todo plan de formación incluya ese componente, con mirada de futuro, porque es un campo que está cambiando vertiginosamente. (C. Lépiz, comunicación personal, 8 de octubre, 2013)

Aquí la cuestión clave en términos de mejorar el Plan de Estudios, como lo explicaba una de las entrevistadas, es "que los estudiantes y la administración [del posgrado] dejen de ver el currículo como algo fragmentado, y se le pueda dedicar más tiempo a una formación realmente integral" (S. Jiménez, comunicación personal, 10 de abril de 2013). Aunque para esto, y haciendo eco de las palabras de uno de los colaboradores, también se requiere "la disponibilidad de un núcleo básico (masa crítica) de profesionales y académicos permanente a nivel de posgrado (...) en docencia universitaria (...) porque es notoria la rotación de personal docente y directivo" (C. Méndez, comunicación personal, 10 de octubre de 2013) en la Maestría a lo largo de los años. 
Al respecto, Gimeno-Sacristán (2010) aporta una idea que no puede obviarse para el futuro de la mención en Docencia Universitaria y de su Plan de Estudios: "la calidad del contenido [curricular] hecho realidad es el resultado de un proceso de juego de perspectivas entre la calidad cultural y pedagógica del profesorado, y la de los textos y demás materiales como fuente de información" (p. 28), y especialmente, como factores de la formación.

Por último, la tercer área temática a considerar corresponde a la reivindicación de la pedagogía y los aportes del pensamiento crítico latinoamericano, o dicho de otra manera, al enfoque formativo situado en las realidades socioeducativas, culturales, políticas e históricas de nuestra América. Puesto que ya se abordó este aspecto en el apartado anterior, solo interesa destacar aquí la creciente importancia que, a nivel regional, viene adquiriendo la mirada y el pensamiento propios, desde nuestras realidades múltiples y diversas, como ejes de la formación docente. No como un simple capricho de chovinismo intelectual, sino como una alternativa que se asume para encontrar nuestro lugar en el mundo del siglo XXI.

Del diálogo con los entrevistados se confirma un aspecto medular del diagnóstico curricular del Plan de Estudios: la ausencia cultural y políticamente producida "de teorías pedagógicas que surjan desde el contexto latinoamericano", sea este "América Latina, Centroamérica, Costa Rica", y que permitirían a docentes y estudiantes una experiencia de formación mucho más cercana con respecto de "nuestro propio sentir, nuestro propio saber y conocer" (G. Sánchez, comunicación personal, 19 de setiembre de 2013).

Una problemática como esta solo podría ser resuelta en la medida en que los gestores curriculares -y las instancias de decisión involucradas en la toma de decisiones- comprendan la importancia de vincular más profundamente la filosofía y la pedagogía latinoamericanas a la formación docente, con una visión de equilibrio y apertura frente a otras corrientes de pensamiento. Es decir, un enfoque de formación de docentes que haga posible, en el campo del currículo, aquella hermosa tesis del cubano José Martí: que se injerte el mundo en nuestras repúblicas, pero que el tronco siga siendo nuestro. 


\section{Referencias bibliográficas}

Apple, M. (2008). Ideología y currículo. Madrid: Ediciones Akal.

Florez, R. (1994). Hacia una pedagogía del conocimiento. Santafé de. Bogotá, Mc Graw Hill.

Garrón, V. (1971). Joaquín García Monge. San José, C.R.: Ministerio de Cultura, Juventud y Deportes.

Gimeno-Sacristán, J. (comp.) (2010). Saberes e incertidumbres sobre el currículo. Madrid: Ediciones Morata.

Maestría en Educación - MED, (1998). Propuesta Mención en Docencia Universitaria (Documento impreso). Heredia, C.R.: División de Educología - CIDE.

Mora, A. (2014). La construcción cultural del currículo: un análisis del Plan de Estudios de la Maestría en Educación con énfasis en Docencia Universitaria de la División de Educología del CIDE, desde una perspectiva crítica latinoamericana. (Tesis de Maestría no publicada). Universidad Nacional, Heredia, Costa Rica.

Mora, A. (en prensa). La construcción cultural del currículo: reflexiones en clave latinoamericana. Ensayos Pedagógicos, IX (2).

Pinto, R. (2008). El currículo crítico. Una pedagogía transformativa para la educación latinoamericana. Santiago de Chile: Ediciones Universidad Católica de Chile.

Pinto, R. (2009). La necesidad de un nuevo paradigma curricular para una época compleja. Revista Posgrado y Sociedad. 9 (1). Pp. 1-25.

Pinto, R. (2012). Principios filosóficos y epistemológicos del ser docente. San José, C.R.: Coordinación Educativa y Cultura Centroamericana / SICA.

Roig, A. (2009). Teoría y crítica del pensamiento latinoamericano. Buenos Aires: Una Ventana.

Sousa, B. (2006). Renovar la teoría crítica y reinventar la emancipación social. Buenos Aires: CLACSO.

Villalobos, T. (2009). Propuesta de rediseño de la Maestría en Educación con Énfasis en Docencia Universitaria (Documento digital). Heredia, C.R.: División de Educología - CIDE. 